\title{
GLIOMAS, TUMORES MALIGNOS QUE SURGEM NO SISTEMA NERVOSO
}

\author{
NEW WEAPONS IN GLIOMA TREATMENT, THE MALIGNANT TUMORS OF THE \\ NERVOUS SYSTEM
}

André Luiz dos Santos ${ }^{1}$

RESUMO: Gliomas são os tumores cerebrais primários mais frequentes, representando mais de $70 \%$ de todas as neoplasias do Sistema Nervoso Central. Tanto o diagnóstico como a classificação destes tumores são baseados nas características histopatológicas que, por vezes, são difíceis de definir. Apesar dos avanços na neurocirurgia, quimioterapia e radioterapia, o prognóstico dos doentes com gliomas continua muito reservado. Nas últimas duas décadas, o tratamento para os gliomas de alto grau envolveu uma abordagem multidisciplinar, que se baseava em resseção cirúrgica máxima, quando possível, seguida de radioterapia e quimioterapia. Esta constitui a abordagem terapêutica convencional, com um grande número de ensaios clínicos que revelaram na sua maioria pequenas melhorias que não são satisfatórias. Sendo assim, este estudo tem como objetivo realizar uma revisão bibliográfica para coletar informações sobre o glioma, assim como, os avanços nos diagnósticos e tratamentos. $\mathrm{Na}$ atualidade as opções terapêuticas disponíveis no tratamento de gliomas, (que incluem, em linhas gerais, cirurgia, RT e QT), ainda não são suficientemente eficazes no controle da progressão da doença. Estudos experimentais demonstraram que a combinação de terapia antiangiogênica, baseada na ruptura de vasos sanguíneos tumorais, com quimioterapia convencional gera resultados encorajadores.

Palavras-chave: Diagnóstico. Tumor Cerebral. Tratamento Terapeutico. Oncologia, Neurobiologia

ABSTRACT: Gliomas are the most common primary brain tumors. It represents a total of $70 \%$ of all Central Nervous System neoplasms. Both the diagnosis and the classification of these tumors are based on hispathological features, which are often difficult to define. Despite advances in the field of neurosurgery, chemotherapy and radiotherapy, the prognosis of glioma patients remains very reserved. Over the past two decades, treatment for high-grade gliomas has involved a multidisciplinary approach that relies on maximum surgical resection (when possible), followed by radiotherapy and chemotherapy. This is the conventional therapeutic approach, with a large number of clinical trials showing mostly minor improvements that cannot be considered satisfactory. Thus, this study aims to conduct a literature review with the objective of collecting information on gliomas, as well as advances in diagnosis and treatments. Current options available for glioma treatment are still insufficient and ineffective in controlling disease progression. Encouraging results have appeared in recent experimental studies. They show that

\footnotetext{
1 Biólogo e Pedagogo, associado profissionalmente ao Banco de Tecidos do Instituto Nacional de Traumatologia e Ortopedia (INTO), como Servidor na Fundação Saúde do Rio de Janeiro (FSERJ). Associado ao Instituto Brasileiro de Medicina de Reabilitação (IBMR) como aluno. E-mail: andrevalente.rio@gmail.com
} 
the combination of antiangiogenic therapy based on tumor vessel rupture and conventional chemotherapy present satisfactory and unusual results!

Keywords: Diagnosis. Brain Tumor. Therapeutic Treatment. Oncology. Neurobiology.

\section{INTRODUÇÃO}

Os tumores no sistema nervoso central (SNC), de forma mais genérica, são classificados em tumores do tecido neuroepitelial (incluem os gliomas), em tumores germinativos (incluem os meduloblastomas e os neuroblastomas), em tumores dos nervos periféricos e em meningiomas, entre outros (LOUIS et alia, 2007). Glioma é o termo utilizado para designar tumores com origem nas células da glia (astrócitos, oligodendrócitos, microglia e ependimárias). Segundo Alves (2014) os principais gliomas cerebrais de alto grau são o astrocitoma anaplásico (AA) e o glioblastoma multiforme (GBM).

Os gliomas fazem parte do grupo dos tumores neuroepiteliais e correspondem a $33 \%$ dos tumores primários e 79\% dos tumores malignos do SNC. O grupo dos astrocitomas corresponde a 75\% de todos os gliomas, e o glioblastoma representa 51,7\% dos casos (OSTROM et alia, 2010). O glioblastoma é o mais frequente e maligno dos astrocitomas e, apesar de inúmeros avanços no diagnóstico e tratamento desses tumores, seu prognóstico permanece ainda bastante limitado (BRANDES et alia, 2008; WEN e KESARI, 2008).

Atualmente, o diagnóstico destes tumores é baseado em estudos de imagem e, para um diagnóstico mais preciso, é requerido tecido tumoral para uma análise histológica. Este tecido é obtido através da biópsia ou resseção o que representa um risco quando os gliomas estão localizados em zonas de difícil acesso (GASPAR, 2016).

A abordagem tradicional para tratar gliomas engloba cirurgia, radioterapia e quimioterapia. A cirurgia é a abordagem terapêutica inicial comum para a resseção do tumor e para a obtenção de amostras para diagnóstico. Os doentes submetidos a resseção do tumor têm um prognóstico mais favorável. É durante a cirurgia que se obtém o tecido (amostra) e este deve ser em quantidade suficiente para realizar a análise molecular (STUPP, 2014).

\section{DISCUSSÃO}

\section{I.I Gliomas}

Os gliomas originam no cérebro ou na medula espinal que elevará das pilhas glial. Estas pilhas formam um tecido chamado "glia", pilhas desse nervo das bordaduras para mantê-los no lugar e para apoiar sua função. Ele é um tipo de tumor cerebral comum, podendo elevar diferentes 
tipos de pilhas glial, que incluem astrocytes, oligodendrocytes e pilhas ependymal. Assim o tumor é nomeado de acordo com o tipo de pilha que ele origina (KLEIHUES et alia, 200o).

Portanto, são tumores geneticamente e histologicamente heterogêneo, associado à significativa morbimortalidade (PRADOS et alia, 200o). No entanto, os gliomas têm sido observados no contexto da síndrome do câncer familiar, geralmente associado ao carcinoma da mama, sarcomas de tecidos moles e leucemia (KLEIHUES et alia, 200o).

Entre mais de roo subgrupos histológicos diferentes de tumores cerebrais, os GAG são as entidades mais frequentes, sendo responsáveis por mais de 50\% dos tumores cerebrais primários, dependendo da idade e do país. A incidência do Gliomas de Alto Grau (GAG) tem aumentado nas últimas décadas e tornou-se significativa na população idosa. Apesar de avanços importantes e recentes no entendimento dos mecanismos de base molecular dos GAG, melhorias notáveis na imagiologia, cirurgia, e RT, assim como a descoberta de uma série de novos fármacos promissores, o prognóstico global permanece reservado, com poucas exceções (MIRIMANOFF, 2014).

$\mathrm{Na}$ maioria dos casos, não são associados fatores de risco. Apesar disso é possível identificar a exposição à radiação ionizante como um fator de risco, como nos casos de tratamento anterior por RT (OHGAKI et alia, 2005). Muitos outros fatores foram estudados nos quais se incluem traumatismos cranianos, campos eletromagnéticos, tabaco e mais recentemente o uso de telemóveis. Nenhum foi comprovado como fator de risco para o desenvolvimento GAG (NARAYANAN et alia, 2012).

Tanto as alterações não hereditárias como a irradiação são ocorrências raras, sendo responsáveis por menos de 10\% de todos os gliomas, sugerindo com efeito que anormalidades genéticas complexas combinadas com fatores ambientais desconhecidos predispõem indivíduos ao desenvolvimento de gliomas (HOFER et alia, 2014).

Os gliomas são diferenciados em baixo grau e alto grau consoante o seu prognóstico e o tipo histológico de tumor. Os tumores de grau I e II são referidos como gliomas de baixo grau e os tumores de grau III e IV são referidos como GAG (WELLER, 20ı1; DUNBAR et alia, 20ıo).

Segundo a OMS o estadiamento tumoral tem 4 categorias.

O grau I aplica-se a lesões com baixo potencial proliferativo e com possibilidade de cura após ressecção cirúrgica. Neoplasias de grau II são geralmente infiltrativas e apesar da sua baixa atividade proliferativa usualmente recidivam. Algumas destas neoplasias tendem a progredir para estágios superiores de malignidade. A designação de grau III da OMS é geralmente reservada para lesões com evidência histológica de malignidade, como é o caso dos astrocitomas. Apresentam-se com anaplasia focal ou dispersa e um potencial proliferativo acentuado com aumento da celularidade, atipia nuclear e atividade mitótica evidente. A designação de grau IV pela OMS está associada a polimorfismo celular, atipia nuclear, atividade mitótica, trombose vascular, 
proliferação microvascular e são neoplasias com propensão para necrose tipicamente associadas a uma evolução rápida da doença, tanto no pré como no pós-operatório, com um desfecho quase sempre fatal (NARAYANAN, 2012; LOUIS et alia, 2007).

Tabela I - Graus dos gliomas segundo classificação de tumores do SNC da OMS

\begin{tabular}{|c|c|}
\hline \multicolumn{2}{|l|}{ GRAUS DOS GLIOMAS } \\
\hline \multicolumn{2}{|l|}{ Astrocitomas difusos e Oligodendrogliomas } \\
\hline Astrocitoma difuso, IDH mutante & II \\
\hline Astrocitoma anaplástico, IDH mutante & III \\
\hline Gliobastoma, IDH wildtype & IV \\
\hline Gliobastoma, IDH mutante & IV \\
\hline Oligodendroglioma, IDH mutante e com a codeleção Ip/ıqq & II \\
\hline Oligodendrogliomas anaplásico, IDH mutante e com a codeleção Ip/ı9q & III \\
\hline \multicolumn{2}{|l|}{ Outros Astrocitomas } \\
\hline Astrocitoma pilocítico & I \\
\hline Astrocitoma subependimário de células gigantes & I \\
\hline Xantoastrocitoma pleomórfico & II \\
\hline Xantoastrocitoma pleomórfico anaplásico & III \\
\hline \multicolumn{2}{|l|}{ Ependimonas } \\
\hline Subependimonas & I \\
\hline Ependimonas mixopapilar & I \\
\hline Ependimona & II \\
\hline Ependimonas anaplásico & III \\
\hline
\end{tabular}

Fonte: OMS, 2016 [adaptado de Louis et al (2016)]

Os gliomas são divididos em subgrupos baseados na morfologia histológica e similaridade das suas células com células da glia diferenciadas (ver tabela I). Desta forma, são classificados, segundo a Organização Mundial de Saúde (OMS), em astrocitomas, oligodendrogliomas e oligoastrocitomas, sendo estes, os três maiores subgrupos. Existe outro subgrupo, os ependimomas, que, ocorrem com uma menor frequência (ISOLAN et alia, 2007; KLEIHUES et alia, 2000).

Uma característica marcante dos gliomas é sua infiltração no parênquima cerebral, uma propriedade que prejudica a ressecção cirúrgica completa; consequentemente, esses tumores podem se repetir, resultando em uma alta taxa de mortalidade. Os gliomas invadem ao longo de rotas preferenciais, como aquelas ao longo dos tratos da substância branca e nos espaços perineuronais e perivasculares. Os componentes extracelulares do cérebro e seus parceiros e moduladores desempenham um papel crucial na invasão celular de glioma (FERRER et alia, 2018). 


\subsection{Diagnóstico}

Atualmente, o diagnóstico destes tumores é baseado em estudos de imagem e, para um diagnóstico mais preciso, é requerido tecido tumoral para uma análise histológica. Este tecido é obtido através da biópsia ou resseção o que representa um risco quando os gliomas estão localizados em zonas de difícil acesso. Para além da dificuldade na recolha de tecido, estas zonas podem apresentar resistência à radio e quimioterapia e pode não ser possível realizar uma cirurgia de remoção. Por estas razões, é de extrema importância encontrar novas estratégias que permitam um diagnóstico precoce, um tratamento infalível e uma monitorização mais efectiva (LIANG et alia, 2016).

No entanto, tanto os testes histopatológicos como a neuroimagem são insensíveis e dispendiosos e podem causar hemorragias e danos cerebrais. Desta maneira, é urgente e altamente necessário desenvolver procedimentos menos invasivos para detetar e monitorizar gliomas (QU et alia, 2015).

Tabela 2- Gliomas malignos: epidemiologia e prognóstico (WELLER, 20II)

\begin{tabular}{lrrrr}
\hline & $\begin{array}{r}\text { Incidência } \\
\text { anual por } \\
\text { 100.000 }\end{array}$ & $\begin{array}{r}\text { Sobrevivência } \\
\text { a r ano (\%) }\end{array}$ & $\begin{array}{r}\text { Sobrevivência } \\
\text { a 3 anos (\%) }\end{array}$ & $\begin{array}{r}\text { Sobrevivência } \\
\text { a 5 anos (\%) }\end{array}$ \\
\hline $\begin{array}{l}\text { Astrocitoma anaplásico } \\
\text { Oligodendroglioma }\end{array}$ & 0,41 & 60,3 & 34,7 & 27,4 \\
anaplásico & 0,12 & 79,9 & 59,6 & 49,4 \\
Glioblastoma & 3,19 & 34,6 & 7,3 & 4,8 \\
\hline
\end{tabular}

O diagnóstico e posterior estadiamento dos tumores cerebrais primários segue a classificação da Organização Mundial de Saúde (OMS) (LOUIS et alia, 2007). A incidência anual de gliomas situa-se em 5-6/10o.ooo pessoas (tabela 2).

Os estudos feitos em gliomas, nos últimos anos, têm identificado marcadores de diagnóstico, de prognóstico e preditivos, suscetíveis de ajudarem a definir a classificação histológica. A presença de mutações em genes que codificam a proteína isocitrato desidrogenase i e 2 (IDHr e IDH2) em astrocitomas, oligodendrogliomas e glioblastomas, a codeleção ip/raq em oligodendrogliomas e a hipermetilação da região promotora do gene MGMT em glioblastomas e astrocitomas são considerados dos marcadores mais interessantes em gliomas (GUEDES, 2010).

Os biomarcadores podem ser definidos como qualquer indicador de diagnóstico mensurável que é usado para avaliar o risco ou a presença de determinada doença. Por outras 
palavras, podem ser descritos como características que são objetivamente medidas e avaliadas como indicadores de processos biológicos normais, processos patogênicos e respostas farmacológicas a uma intervenção terapêutica (GASPAR, 2016).

Existem três categorias reconhecidas de biomarcadores. Os biomarcadores de diagnóstico, que identificam a presença de uma doença específica. Os biomarcadores de prognóstico, usados para deduzirem a evolução da doença independentemente da terapia. Finalmente, os biomarcadores preditivos, que informam sobre a resposta a um tratamento particular (BERGHOFF et alia, 2012; REDZIC, J et alia, 2014).

Muitos autores abordam a importância do uso de biomarcadores. Os biomarcadores desempenham um papel importante, por vezes, indispensável na deteç̧ão e monitorização de doentes com malignidades. Assim, em doentes assintomáticos, os biomarcadores podem ser usados na deteç̧ão precoce de cancro ou condições pré-malignas, enquanto em pacientes sintomáticos, os biomarcadores podem ajudar na diferenciação entre doença benigna e maligna. Após um diagnóstico de malignidade, os biomarcadores podem ajudar no prognóstico e na identificação da terapia mais apropriada. Em doentes que tenham sido submetidos a cirurgia de resseção, estes podem ser usados na monitorização e na detecção precoce de possíveis recorrências da doença. E no caso de doentes que estejam a receber tratamento sistêmico, os biomarcadores podem proporcionar uma abordagem menos invasiva para monitorizar a resposta do tumor (DUFFY et alia, 2015; KARSY et alia, 2015).

\section{I.3 Tratamento}

A terapia padrão inclui a ressecção cirúrgica, radioterapia e quimioterapia com administração de temozolomida durante e após a radioterapia. No entanto, o prognóstico é sombrio porque a maioria dos pacientes desenvolve recorrência desses tumores e ou, progressão após este tratamento multimodal (LOUIS et alia, 2007; SIQUEIRA et alia, 1999).

$\mathrm{Na}$ atualidade as opções terapêuticas disponíveis no tratamento de gliomas, (que incluem, em linhas gerais, cirurgia, RT e QT), ainda não são suficientemente eficazes no controlo da progressão da doença. A sobrevida máxima é alcançada nos doentes que são submetidos a uma resseção total, seguida de RT e Tmz, mas a sobrevida média nesta população continua a ser de apenas 20 meses (WOLBERS, 2014).

O ensaio preponderante da Organização Europeia de Pesquisa e Tratamento do Cancro juntamente com o Instituto Nacional de Cancro do Canadá (OEPTC-INCC), publicado em 2005 e atualizado em 2009, estabeleceu o tratamento estandardizado para GBM (STUPP et alia, 2005; STUPP et alia, 2009). Apesar de uma minoria significativa de pacientes sobreviver vários anos 
(HEGI et alia, 2005; MIRIMANOFF et alia, 2006), a maioria sucumbe à doença. Progresso também foi feito no que toca a AA e AO (CAIRNCROSS et alia, 2or3; VAN DEN BENT et alia, 2013), para os quais o prognóstico é menos sombrio. A qualidade de vida relacionada com a saúde é uma ferramenta de importância crescente nos GAG (TAPHOORN et alia, 2005; TAPHOORN et alia, 2010), devendo ser um aspeto a incluir em qualquer ensaio prospetivo, de forma a facilitar a decisão terapêutica final.

A abordagem com melhores resultados em relação aos GAG envolve uma equipa multidisciplinar. As opções para essa abordagem vão desde o tratamento conservador à cirurgia e terapia adjuvante. O principal objetivo é o diagnóstico e o prolongamento da sobrevida sem intercorrências. A dexametasona tem uma grande importância no controlo rápido do edema cerebral associado aos tumores. Uma eventual ineficácia dos esteróides sugere um maior défice neurológico no caso de uma resseção cirúrgica radical (STUMMER, 20II). As terapêuticas convencionais permanecem como os principais meios disponíveis para fazer face aos GAG.

Uma das formas mais antigas e, quiçá, ainda a mais importante no tratamento disponível para pacientes com glioma, é a cirurgia. Mesmo no meio contemporâneo de regimes multimodais, incluindo RT e QT, a resseção do glioma continua a ser um esteio (CHOI et alia, 2012).

Uma alternativa para a Quimioterapia sistêmica envolve a implantação cirúrgica de polímeros biodegradáveis contendo carmustina (Gliadel ${ }^{\circledR}$ Wafer) na cavidade de resseção tumoral. Um estudo randomizado, duplo cego de fase III demonstrou um benefício modesto em pacientes com GBM recém-diagnosticados. Os pacientes que receberam radiação e placebo obtiveram uma sobrevida de apenas II.6 meses, em comparação com os 13.9 meses de sobrevida para os pacientes que receberam radiação e carmustina (WESTPHAL et alia, 2003).

O tratamento clássico do glioma anaplásico recém-diagnosticado inclui uma resseção cirúrgica total se possível, seguida de Radioterapia (RT) (54-6o Gy em frações de r.8-2 Gy) diretamente sobre a região do tumor com uma margem de segurança de $2-3 \mathrm{~cm}$, dependendo da localização. A cirurgia em pacientes com gliomas malignos tem como objetivo a remoção da maior quantidade possível do tumor, sem causar défices neurológicos. A biópsia é possivelmente a única opção para tumores multifocais ou que envolvam o corpo caloso. O papel da QT em adição à RT tem sido examinado em inúmeros estudos, mas os resultados são inconclusivos. Foi realizado um estudo britânico de grandes dimensões para avaliar a combinação de procarbazina, lomustina e vincristina, ainda que em dosagens incomuns, tendo sido obtida uma sobrevida média de 15 meses com a RT + vincristina versus 13 meses apenas com RT (PARTY, 200I). 
Terapias moleculares específicas são uma perspectiva interessante, atualmente em vários estágios de pesquisa e desenvolvimento. Estes são fármacos que têm como objetivo atingir as vias oncogênicas em gliomas, através da interação com os receptores ou afetando um alvo a jusante. Os ensaios clínicos de fármacos que bloqueiam o receptor do fator de crescimento epidérmico (RFCE), o receptor do fator de crescimento derivado de plaquetas (RFCDP) e o fosfatidilinositol 3-quinase $\left(\mathrm{PI}_{3} \mathrm{~K}\right)$ e respectivas vias, até agora têm sido dececionantes (NARAYANAN, 20I2).

Alves (2014) aborda que com os inúmeros estudos e ensaios realizados até à data, é unanimemente reconhecido que será necessária uma abordagem combinada para a obtenção de um prognóstico mais favorável com o respectivo aumento da sobrevida dos pacientes, essa abordagem poderá passar pelo uso de Nanopartículas para marcar as células tumorais seguido da $\mathrm{RT}$ e QT convencionais.

Ainda de acordo com outros autores há estudos sobre o uso de um tratamento imunológico. O glioblastoma (GBM) é o tumor cerebral maligno primário mais comum e fatal. Apesar dos avanços no entendimento da biologia dos gliomas, pouco mudou no tratamento desses tumores na última década. Os ensaios clínicos de fase III não mostraram benefício para o uso de bevacizumabe em pacientes recém-diagnosticados, levando a uma busca renovada por novos medicamentos antiangiogênicos, bem como abordagens imunoterapêuticas, incluindo inibidores de ponto de verificação, células $\mathrm{T}$ do receptor de antígeno quimérico e $\mathrm{CpG}$ oligodesoxinucleotídeos intracerebrais. $O$ papel emergente da infiltração de micróglias e macrófagos e de alterações metabólicas também está sendo levado em consideração na pesquisa pré-clínica e no desenvolvimento de medicamentos (GERALDO et alia, 2018).

Estudos experimentais demonstraram que a combinação de terapia antiangiogênica, baseada na ruptura de vasos sanguíneos tumorais, com quimioterapia convencional gera resultados encorajadores. Relatórios emergentes também mostraram que as células microgliais podem ser usadas como vetores terapêuticos para transportar genes e / ou substâncias para o local do tumor, o que abre novas perspectivas para o desenvolvimento de terapias GBM direcionadas às células microgliais. Finalmente, estudos recentes mostraram que as toxinas naturais podem ser conjugadas com drogas que se ligam a receptores superexpressos nas células cancerígenas, gerando toxinas direcionadas para matar seletivamente as células cancerígenas. Essas toxinasalvo são altamente eficazes contra células cancerígenas resistentes à radiação e quimioterapia, tornando-as boas candidatas a ensaios clínicos em pacientes com GBM (LIMA et alia, 2012). 


\section{Discussão}

Os gliomas são o tipo mais comum de tumor cerebral primário, compreendendo cerca de $50 \%$ de tumores cerebrais malignos em adultos. São assim denominados porque têm origem em células da glia e são subdividos em astrocitomas, oligodendrogliomas e ependimomas com base na similaridade no perfil imunofenotípico das células quem lhes dão origem. Tumores que exibam uma mistura de diferentes células são denominados oligoastrocitomas.

Atualmente, o diagnóstico destes tumores é baseado em estudos de imagem e, para um diagnóstico mais preciso, é requerido tecido tumoral para uma análise histológica. Este tecido é obtido através da biópsia ou resseção o que representa um risco quando os gliomas estão localizados em zonas de difícil acesso. Os biomarcadores circulantes surgem então como um meio promissor e não-invasivo para avaliar o estado dos gliomas e, potencialmente, ajudar no seguimento e monitorização da terapia no futuro. $\mathrm{Na}$ atualidade as opções terapêuticas disponíveis no tratamento de gliomas, (que incluem, em linhas gerais, cirurgia, RT e QT), ainda não são suficientemente eficazes no controlo da progressão da doença. Estudos experimentais demonstraram que a combinação de terapia antiangiogênica, baseada na ruptura de vasos sanguíneos tumorais, com quimioterapia convencional gera resultados encorajadores.

\section{Referências}

ALVES, P.M.G. Abordagem Terapêutica de Gliomas Cerebrais de Alto Grau. Dissertação (Mestrado em Medicina) - Universidade da Beira Interior, Covilhã, 2014.

BERGHOFF, A. et alia. Clinical Neuropathology Practice News 4-2012: levels of evidence for brain tumor biomarkers. Clinical neuropathology. ISSN 0722-5091. 31:4 206-9.

BRANDES A.A. et alia. Glioblastoma in adults. Critical Reviews in Oncology/Hematology 67:139-152. Disponível em <https://www.pennstatehershey.org/c/document_library/get_file?uuid=e7090489-of27-46a7803c-e8db5d2191eb\&groupId=101847>, Acesso em: 20 out. 2019.

CAIRNCROSS, G. et alia. Phase III trial of chemoradiotherapy for anaplastic oligodendroglioma: long-term results of RTOG 9402. Journal of Clinical Oncology, 2013. 3I(3): p. $337-43$.

CHOI, B.D. et alia. The use of motor mapping to aid resection of eloquent gliomas. Neurosurgery Clinics of North América, 2012. 23(2): p. 215-25, vii.

DUFFY, M. J. et alia. Validation of new cancer biomarkers: a position statement from the European group on tumor markers. Clinical chemistry. ISSN 1530-8561. 61:6 (2015) 809-20.

DUNBAR, E. and A.T. Yachnis, Glioma diagnosis: immunohistochemistry and beyond. Adv Anat Pathol, 2010. 17(3): p. 187-201. 
FERRER, V. P. et alia. Glioma infiltration and extracellular matrix: key players and modulators. GLIA, v. 2, p. I-24, 2018.

GASPAR, B. M. Biomarcadores em Gliomas: Conhecimento Atual e Perspetivas Futuras. Dissertação (Mestrado Integrado em Ciências Farmacêuticas), Faculdade de Farmácia da Universidade de Coimbra, Coimbra, 2016.

GERALDO, L.H.M. et alia. Glioblastoma Therapy in the Age of Molecular Medicine. Trends in Cancer, Volume 5, Issue I, Janeiro de 2019, p. 46-65. Disponível em 〈https://www.sciencedirect.com/science/article/abs/pii/S2405803318302553〉, Acesso em: 20 out. 2019.

GUEDES, A. Estudo de marcadores de diagnóstico e prognóstico em gliomas. 2010. II6 f. Dissertação (Mestrado em Biotecnologia para as Ciências da Saúde) - Universidade de Trás-osMontes e Alto Douro, Vila Real, 2010.

HEGI, M.E. et alia. MGMT gene silencing and benefit from temozolomide in glioblastoma. The New England Journal of Medicine, 2005. 352(10): p. 997-1003.

HOFER, S., et alia, Molecular biology of high-grade gliomas: what should the clinician know? Chinese Journal of Cancer Research, 2014. 33(I): p. 4-7.

ISOLAN GR, et alia. Understanding the astrocytic neo-plasm stumorigenesis. Revista Médica do Paraná, Curitiba, 2007.

KARSY, M. et alia. A practical review of prognostic correlations of molecular biomarkers in glioblastoma. Neurosurgical focus. ISSN 1092-0684. 38:3 (2015) E4.

KLEIHUES P, et alia. World Health Organization classification of tumors. Cancer, ActaNeuropathol, 2000.

LIANG, S. e SHEN, G. Biomarkers of Glioma. Disponível em 〈http://cdn.intechopen.com/pdfs/19940/InTech-Biomarkers_of_glioma.pdf 〉, Acesso em: 20 out. 2019.

LIMA, F.R.S. et alia. Glioblastoma: Therapeutic challenges, what lies ahead. Biochimica et Biophysica Acta, Reviews on Cancer, Volume 1826, Issue 2, Dezembro de 2012, p. 338-349. Disponível em 〈https://www.sciencedirect.com/science/article/abs/pii/So304419XI2000443?via\%3Dihub〉, Acesso em: 20 out. 2019.

LOUIS, D.N., et alia, The 2007 WHO classification of tumours of the centralnervous system. Acta Neuropathol, 2007;114(2): 97-109.

LOUIS, D.N., et alia, The 2007 WHO classification of tumours of the central nervous system. Acta Neuropathol, 2007. II4(2): p. 97-109.

MIRIMANOFF, R.O. et alia. Radiotherapy and temozolomide for newly diagnosed glioblastoma: recursive partitioning analysis of the EORTC 2698I/2298I-NCIC CE3 phase III randomized trial. Journal of Clinical Oncology, 2006. 24(16): p. 2563-9. 
MIRIMANOFF, R.O., High-grade gliomas: reality and hopes. Chinese Journal of Cancer Research, 2014. 33(I): p. I-3.

NARAYANAN V. High Grade Gliomas: Pathogenesis, Management and Prognosis. Advances in Clinical Neuroscience and Rehabilitation, 2012. 12: p. 24-29.

OHGAKI, H.; P. Kleihues, Epidemiology and etiology of gliomas. Acta neuropathologica, 2005. IO9(I): p. 93-108.

OSTROM, Q.T. et alia. CBTRUS Statistical Report: Primary brain and other central nervous system tumors diagnosed in the United States in 2010-2014. Disponível em 〈https://academic.oup.com/neuro-oncology/article/rig/suppl_5/vi/4596648〉, Acesso em: 20 out. 2019 .

PARTY, M.R.C.B.T.W. Randomized trial of procarbazine, lomustine, and vincristine in the adjuvant treatment of high-grade astrocytoma: a Medical Research Council trial. Journal of Clinical Oncology, 2001. 19(2): p. 509-18.

PRADOS M.D. e LEVIN V. Biology and treatment of malignant glioma. Seminars in Oncology, 3I May 2000.

QU, Shengtao et alia. Identification of microRNAs as novel biomarkers for glioma detection: A meta-analysis based on II articles. Journal of the Neurological Sciences. ISSN I8785883. 348:1-2 (2015) $18 \mathrm{I}-187$

REDZIC, J. S et alia. Glioblastoma extracellular vesicles: reservoirs of potential biomarkers. Pharmacogenomics and personalized medicine. ISSN i178-7066. 7: (2014) 65-77.

SIQUEIRA, M.G. et alia. Biologia, Diagnóstico e Tratamento: Tumores Intracranianos. Rio de Janeiro: Revinter, I999.

STUMMER, W. et alia. Counterbalancing risks and gains from extended resections in malignant glioma surgery: A supplemental analysis from the randomized 5-aminolevulinic acid glioma resection study. Clinical study. Journal of neurosurgery, 2011. 114. 613-23. 10.3171/2010.3.JNSo97.

STUPP, R. et alia. Effects of radiotherapy with concomitant and adjuvant temozolomide versus radiotherapy alone on survival in glioblastoma in a randomised phase III study: 5-year analysis of the EORTC-NCIC trial. Lancet Oncol, 2009. Io(5): p. 459-66.

STUPP, R. et alia. High-grade glioma: ESMO Clinical Practice Guidelines for diagnosis, treatment and follow-up. Annals of Oncology. ISSN 0923-7534. 25:suppl 3 (2014) iiig3-iiiror.

STUPP, R. et alia. Radiotherapy plus concomitant and adjuvant temozolomide for glioblastoma. The New England Journal of Medicine, 2005. 352(ro): p. 987-96.

TAPHOORN, M.J. et alia. An international validation study of the EORTC brain cancer module (EORTC QLQ-BN20) for assessing health-related quality of life and symptoms in brain cancer patients. Eur J Cancer, 2010. 46(6): p. 1033-40. 
TAPHOORN, M.J. et alia. Health-related quality of life in patients with glioblastoma: a randomised controlled trial. Lancet Oncol, 2005. 6(12): p. 937-44.

VAN DEN BENT, M.J. et alia. Adjuvant procarbazine, lomustine, and vincristine chemotherapy in newly diagnosed anaplastic oligodendroglioma: long-term follow-up of EORTC brain tumor group study 26951. Journal of Clinical Oncology, 2013. 3I(3): p. 344-50.

WELLER, M., Novel diagnostic and therapeutic approaches to malignant glioma. Swiss Med Wkly, 20II. I4I: p. wi3210.

WEN PY, KESARI S. Malignant gliomas in adults. The New England Journal of Medicine. 2008;359(5):492-507.

WESTPHAL, M., et alia. A phase 3 trial of local chemotherapy with biodegradable carmustine (BCNU) wafers (Gliadel wafers) in patients with primary malignant glioma. Neuro Oncol, 2003. 5(2): p. $79-88$.

WOLBERS, J.G. Novel strategies in glioblastoma surgery aim at safe, supra-maximum resection in conjunction with local therapies. Chinese Journal of Cancer Research, 2014. 33(I): p. 8-15. 